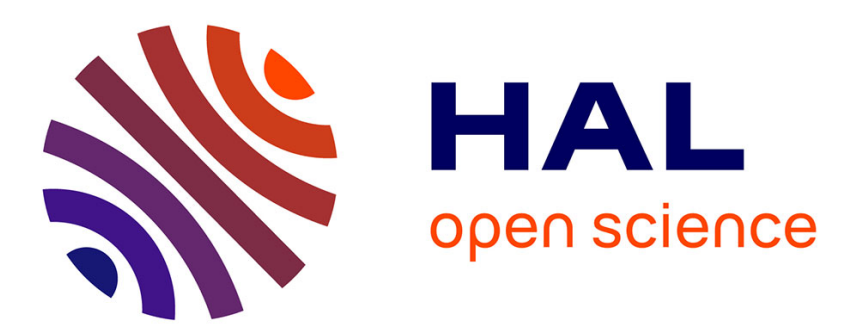

\title{
Practical identifiability of photophysical parameters in photodynamic therapy
}

Simona Dobre, Thierry Bastogne, Muriel Barberi-Heyob, Alain Richard

\section{To cite this version:}

Simona Dobre, Thierry Bastogne, Muriel Barberi-Heyob, Alain Richard. Practical identifiability of photophysical parameters in photodynamic therapy. 29th Annual International Conference of the IEEE Engineering in Medicine and Biology Society, EMBC'2007, Aug 2007, Lyon, France. pp.CDROM. hal-00167475

\section{HAL Id: hal-00167475 \\ https://hal.science/hal-00167475}

Submitted on 20 Aug 2007

HAL is a multi-disciplinary open access archive for the deposit and dissemination of scientific research documents, whether they are published or not. The documents may come from teaching and research institutions in France or abroad, or from public or private research centers.
L'archive ouverte pluridisciplinaire HAL, est destinée au dépôt et à la diffusion de documents scientifiques de niveau recherche, publiés ou non, émanant des établissements d'enseignement et de recherche français ou étrangers, des laboratoires publics ou privés. 


\title{
Practical identifiability of photophysical parameters in photodynamic therapy
}

\author{
Simona Dobre*, Thierry Bastogne*,Muriel Barberi-Heyob ${ }^{\dagger}$, Alain Richard* \\ *Centre de Recherche en Automatique de Nancy (CRAN), Nancy - Université, CNRS UMR 7039, \\ BP 239, F-54506 Vandœuvre-lès-Nancy Cedex, France, \\ Simona.Dobre; Thierry. Bastogne; Alain.Richardecran.uhp-nancy. fr \\ $\dagger$ Centre de Recherche en Automatique de Nancy (CRAN), Nancy - Université, CNRS UMR 7039, \\ Centre Alexis Vautrin, 54511 Vandœuvre-lès-Nancy Cedex, France, \\ m.barberi@nancy.fnclcc.fr
}

\begin{abstract}
Photodynamic therapy (PDT) is an alternative treatment for cancer that involves the administration of a photosensitizing agent, which will be activated by light at a specific wavelength. In order to compare different photosensitisers for their cytotoxic activity, photophysical parameters provide good indicators. These parameters are generally estimated one by one from in vitro dedicated experiments, but they cannot always predict the in vivo cytotoxic action. So far, the estimation of photophysical parameters from in vivo data sets has never been regarded as a system identification problem. This paper deals with the practical identifiability of photophysical parameters. Practical identifiability deals with the uniqueness of the model parameters estimates, given the experimental data. The practical identifiability approach and its application to the photoreaction model of PDT are developed in this paper. It is shown that that the photophysical parameters involved in the kinetic model of photoreactions are identifiable in a practical framework with only one measurement - the intracellular photosensitizer concentration, and a wide square pulse as irradiation signal.
\end{abstract}

\section{INTRODUCTION}

Photodynamic therapy (PDT) [1], [2], [9] is a therapy for displastic tissues such as cancers. This therapy involves selective uptake and retention of a photosensitive drug (photosensitiser, PS) in a tumor, followed by irradiation with light at an appropriate wavelength. The activatation of the photosensitiser is thought to produce singlet oxygen at high doses and thereby to initiate apoptotic and necrotic death of tumour.

For the PDT application, many different photosensitisers have been developed. It is important to compare these photosensitisers for their cytotoxic activity. It is generally accepted that the most important mechanism of cell killing is via the production of singlet oxygen. Currently, these photosensitisers are compared upon some photophysical properties: (1) the absorption coefficient, $\sigma_{S}$ of the PS in the spectral region of the excitation light; (2) the quantum yield of the triplet state, $\Phi_{T}$; (3) the triplet state lifetime $\tau_{T}$ and (4) the quantum yield of singlet oxygen production, $\Phi_{\Delta}$. Most of the time, these parameters are determined from dedicated in vitro experiments, and unfortunately are rarely determined from in vivo conditions. So far, few studies

\footnotetext{
${ }^{1}$ Communication presented in the 29th International Conference of the IEEE Engineering in Medicine and Biology Society, Lyon, France, 2007
}

have reported some developments in the measurement of the singlet oxygen concentration through the detection of its luminescence intensity at $1272 \mathrm{~nm}$ [3], [4]. Moreover, these developments are still difficult to reproduce in practice, due in particular to the interlesion and interpatient variations. This paper proposes an alternative approach.

A model of the photoreaction phase is introduced, based on the kinetics equations describing the type-II reactions specific to PDT. The singlet oxygen yield explicitly depends on photophysical parameters of these photoreactions. In other terms, these photophysical parameters provide good indicators to compare the cytotoxic activities of PS. So far, the estimation of photophysical parameters from in vivo data sets has never been regarded as a system identification problem [11]. The objective of this paper is thus to assess the practical identifiability of photophysical parameters in PDT by using an approach recently reported in [5]. The latter approach requires the dynamic model to be expressed as a block diagram implemented into the simulation environment Simulink ${ }^{\circledR}$ for the symbolic computation of the sensitivity functions.

\section{Modeling OF PHOTOREACtions}

This part is dedicated to the modelling of the main reactions involved in the mechanism of singlet oxygen production. A summary of the notations used in the sequel is given in Tab. I, and a list of all the reactions considered in this modelling study is given in Tab. II. Considering the reactions presented in Tab. II and adopting the notations in Tab. I, the photoreaction phase can be described by a nonlinear statespace model, defined as follows

$$
\left\{\begin{array}{l}
\dot{\mathbf{x}}=f(\mathbf{x}, \mathbf{u}, t, \Theta) \\
y=\gamma \cdot\left[S_{0}\right] \\
\mathbf{x}(0)=\mathbf{x}_{0}
\end{array}\right.
$$

where $\mathbf{x}, \mathbf{u}$ and $y$ denote the state vector, the input vector and the output variable (fluorescence intensity) respectively, with $\mathbf{x}=\left(\left[S_{0}\right],\left[S_{1}\right],\left[T_{1}\right],\left[{ }^{3} O_{2}\right],\left[{ }^{1} O_{2}\right],[M]\right)^{T}$ and $\mathbf{u}=\left(V_{A}, U_{P}, U_{O_{2}}\right)$. $\mathbf{x}_{0}$ denotes the initial value of the state variable and $t$ is the time variable. $f(\cdot)$ contains the state equations and $\gamma$ is the gain of the measurement system (spectrofluorimeter). All the photophysical parameters are gathered in $\Theta$. The 
TABLE I

NOTATIONS

\begin{tabular}{|c|c|c|}
\hline Symbol & Definition & Units \\
\hline$V_{A}$ & Rate of photon absorption & $M \cdot s^{-1}$ \\
\hline$U_{P}$ & Uptake rate of photosensitising molecules & $M \cdot s^{-1}$ \\
\hline$U_{O_{2}}$ & Uptake rate of oxygen molecules & $M \cdot s^{-1}$ \\
\hline$\Phi_{i}$ & Irradiance of the incident light & $\mathrm{mW} \cdot \mathrm{cm}^{-2}$ \\
\hline$\left[S_{0}\right]$ & Photosensitizer ground state & $M$ \\
\hline$\left[S_{1}\right]$ & Photosensitizer singlet excited state & $M$ \\
\hline$\left[T_{1}\right]$ & Photosensitizer triplet excited state & $M$ \\
\hline${ }^{3} O_{2}$ & Triplet ground - state oxygen & $M$ \\
\hline${ }^{1} O_{2}$ & Singlet excited - state oxygen & $M$ \\
\hline$[M]$ & Cellular targets & $M$ \\
\hline
\end{tabular}

TABLE II

SUMMARY OF PHOTOCHEMICAL REACTIONS

\begin{tabular}{|l|c|c|}
\hline Photochemical Reaction & Rate Constant & Units \\
\hline $1 . S_{0}+h v_{A} \rightarrow S_{1}$ & $V_{A}$ & $M \cdot s^{-1}$ \\
\hline 2. $S_{1} \rightarrow S_{0}+h v_{F}$ & $k_{F}$ & $s^{-1}$ \\
3. $S_{1} \rightarrow S_{0}$ & $k_{I C}$ & $s^{-1}$ \\
4. $S_{1} \rightarrow T_{1}$ & $k_{I S C}$ & $s^{-1}$ \\
\hline 5. $T_{1} \rightarrow S_{0}+h v_{P}$ & $k_{P}$ & $s^{-1}$ \\
6. $T_{1} \rightarrow S_{0}$ & $k_{T S}$ & $s^{-1}$ \\
\hline 7. $T_{1}+{ }^{3} O_{2} \rightarrow S_{0}+{ }^{1} O_{2}$ & $k_{T}$ & $M^{-1} \cdot s^{-1}$ \\
\hline 8. ${ }^{1} O_{2}+S_{0} \rightarrow{ }^{3} O_{2}+S(O)$ & $k_{P b}$ & $M^{-1} \cdot s^{-1}$ \\
\hline 9. ${ }^{1} O_{2} \rightarrow{ }^{3} O_{2}+h v_{L}$ & $k_{r}$ & $s^{-1}$ \\
10. ${ }^{1} O_{2} \rightarrow{ }^{3} O_{2}$ & $k_{n r}$ & $s^{-1}$ \\
\hline $11 .{ }^{1} O_{2}+M \rightarrow{ }^{3} O_{2}+M(O)$ & $k_{O x}$ & $M^{-1} \cdot s^{-1}$ \\
\hline
\end{tabular}

state equations corresponding to the photoreactions defined in Tab. II are

$$
\left\{\begin{array}{l}
\frac{d\left[S_{0}\right]}{d t}=U_{P}(t)+\left(k_{F}+k_{I C}\right)\left[S_{1}\right]-k_{P b}\left[{ }^{3} O_{2}\right]\left[S_{0}\right] \\
+\left(k_{P}+k_{T S}\right)\left[T_{1}\right]+k_{T}\left[T_{1}\right]\left[{ }^{3} O_{2}\right]-V_{A}(t) \\
\frac{d\left[S_{1}\right]}{d t}=V_{A}(t)-\left(k_{F}+k_{I C}+k_{I S C}\right)\left[S_{1}\right] \\
\frac{\left.d T_{1}\right]}{d t}=k_{I S C}\left[S_{1}\right]-\left(k_{P}+k_{T S}\right)\left[T_{1}\right]-k_{T}\left[T_{1}\right]\left[{ }^{3} O_{2}\right] \\
\frac{\left.d^{3} O_{2}\right]}{d t}=U_{O_{2}}-k_{T}\left[T_{1}\right]\left[{ }^{3} O_{2}\right]+\left(k_{r}+k_{n r}\right)\left[{ }^{1} O_{2}\right] \\
\frac{d\left[{ }^{1} O_{2}\right]}{d t}=k_{T}\left[T_{1}\right]\left[{ }^{3} O_{2}\right]-\left(k_{r}+k_{n r}\right)\left[{ }^{1} O_{2}\right] \\
-k_{O x}[M]\left[{ }^{1} O_{2}\right]-k_{P b}\left[S_{0}\right]\left[{ }^{1} O_{2}\right] \\
\frac{d[M]}{d t}=-k_{o x}[M]\left[{ }^{1} O_{2}\right]
\end{array}\right.
$$

The rate of photon absorption, $V_{A}$, depends on the (groundstate) photosensitizer concentration. Conforming to [10], $V_{A}$ can be expressed as

$$
V_{A}=\frac{\sigma_{S} \Phi_{i}}{h v_{A}} \cdot\left[S_{0}\right]
$$

where $\sigma_{S}$ is the absorption cross section of $S_{0}, h$ is the Planck's constant and $v_{A}$ is the frequency of the incident light. Therefore, the vector of parameters is given by

$$
\Theta^{T}=\left(\begin{array}{lllllll}
k_{f} & k_{p} & k_{T} & k_{P b} & k_{I S C} & k_{l} & k_{o x}
\end{array}\right)
$$

with $k_{f}=k_{F}+k_{I C}, k_{p}=k_{P}+k_{T S}$ and $k_{l}=k_{r}+k_{n r}$.

The relationship between these parameters and the quan- tum yield of singlet oxygen production, $\Phi_{\Delta}$, is

$$
\Phi_{\Delta}=\Phi_{T} \cdot \phi_{e n}=\Phi_{T} \cdot\left(\frac{k_{T}\left[{ }^{3} O_{2}\right]}{k_{P}+k_{T S}+k_{q}\left[{ }^{3} O_{2}\right]}\right)
$$

where $\Phi_{T}$ is the quantum yield of triplet formation; $\phi_{e n}$, efficiency of energy transfer and $k_{q}$ is the sum of rate constants from the quenching of $T_{1}$ by $O_{2}$ (knowing that bimolecular reactions such as physical deactivation by molecular oxygen or electron transfer can also compete with energy transfer).

Therefore, determining the parameter values from in vivo conditions, we could compare different photosensitizers.

\section{Problem Statement}

Consider $\mathbf{x}(t, \Theta) \in \mathbb{R}^{n}, y(t, \Theta) \in \mathbb{R}, \Theta \in \mathbb{P}$ and $\mathbf{u}(t) \in \mathbb{U}$. $\mathbb{P}$ and $\mathbb{U}$ are two open sets in $\mathbb{R}^{p}$ and $\mathbb{R}^{q}$ respectively with $n=6, p=7$ and $q=3$. $\mathbb{T}$ is a set of time instants defined by $\mathbb{T}=\left\{t_{j}\right\}, j=0, \cdots, N-1 . N, p, q, n$ denote the numbers of observations, parameters, input variables and state variables respectively. $\mathbf{x}_{0}=x\left(t_{0}, \Theta\right)$ is the fixed known initial state. Now suppose that a data set $\{\mathbf{u}(t), y(t)\}$, with $t \in \mathbb{T}$, resulting from one experiment, is available. The classical identification problem consists in estimating $\Theta$ from the observed data (u and y). However, by answering the investigated question, remains to know if whether or not the in vivo data $\{\mathbf{u}, y\}$ may be used to estimate the parameters of the model. The associated underlying problem is the practical identifiability of photophysical parameters.

\section{PRACTICAL IDENTIFIABILITY}

\section{A. Method}

Structural identifiability [11] deals with the possibility to give a unique value to each parameter of a mathematical model structure. The uniqueness of this solution is assessed in an idealized or theoretical framework where the process and the model have identical structures, the data are noisefree, and where the input signals and the measurement times can be chosen at will.

However, in practice, experimental conditions are often subjected to economical and/or technical constraints which can sometimes prevent input design from being applied to the process. Moreover, the number of observations is often limited to a few data points collected at time instants $\left\{t_{j}\right\}, j=0, \cdots, N-1$. In such a case, even if a parameter is globally or locally structurally identifiable, it may not be so in practice, due to a lack of information in the available observations. For that reason, D. Dochain and P. Vanrolleghem, in [13], [14], have introduced the notion of practical identifiability. The practical identifiability includes the quality of the data. The main question of the structural and practical identifiability analysis can then be formulated as follows: 'Assume that a certain number of state variables are available for measurements; on the basis of the model structure (theoretical identifiability) or on the basis of the type and quality of available data (practical identifiability), can we expect to obtain unique values for the model parameters?'. The practical identifiability is just a particular case 
of the output distinguishability [12] for a finite collection of observations $\left\{t_{j}\right\}$ and a given experiment $\left(\mathbf{x}_{0}, \mathbf{u}\right)$. Then, a sufficient condition for the practical identifiability can be stated as follows: given a parametric model structure with given input signals $\mathbf{u}$ and the initial conditions $\mathbf{x}_{0}$,

$$
y\left(t_{j}, \Theta, \mathbf{x}_{0}, \mathbf{u}\right)=y\left(t_{j}, \Theta^{*}, \mathbf{x}_{0}, \mathbf{u}\right) \Rightarrow \Theta_{i}=\Theta_{i}^{*},
$$

$\forall i \in\{1, \cdots, p\}, \forall t_{j} \in \mathbb{T}$ and $\forall \Theta \in V\left(\Theta^{*}\right) \subset \mathbb{P}$. If $d \Theta \in V\left(\Theta^{*}\right)$ with $\Theta=\Theta^{*}+d \Theta$, then a first-order Taylor expansion of $y\left(t, \Theta^{*}+d \Theta, \mathbf{x}_{0}, \mathbf{u}\right)$ is given by

$$
y\left(t, \Theta^{*}+d \Theta, \mathbf{x}_{0}, \mathbf{u}\right) \approx y\left(t, \Theta^{*}, \mathbf{x}_{0}, \mathbf{u}\right)+\left.\sum_{i=1}^{p} \frac{\partial y}{\partial \Theta_{i}}\right|_{\Theta_{i}^{*}} d \Theta_{i} .
$$

$V\left(\Theta^{*}\right)$ denotes a parameter neighbourhood. A local approximation of the practical identifiability condition defined in (6) is then given by

$$
\left.\sum_{i=1}^{p} \frac{\partial y}{\partial \Theta_{i}}\right|_{\Theta_{i}^{*}} d \Theta_{i}=0 \Rightarrow d \Theta=0
$$

or

$$
\sum_{i=1}^{p} d \Theta_{i} \cdot S_{y}\left(t, \Theta_{i}^{*}, \mathbf{x}_{0}, \mathbf{u}\right)=0 \Rightarrow d \Theta=0,
$$

where $S_{y}\left(t, \Theta_{i}^{*}, \mathbf{x}_{0}, \mathbf{u}\right)=\partial y /\left.\partial \Theta_{i}\right|_{\Theta_{i}^{*}}$ denotes the sensitivity function of the model output $y$ related to the parameter $\Theta_{i}$. Equation (9) expresses the linear independence of the vectors $S_{y}\left(t, \Theta_{i}^{*}\right) \in \mathbb{R}^{m}$. In other terms, given the input $\mathbf{u}$ and the initial condition $\mathbf{x}_{0}$, the parameters are locally practically identifiable if the mapping $S_{y}\left(t, \Theta_{i}^{*}, \mathbf{x}_{0}, \mathbf{u}\right)$, from the parameter space to outputs, is one to one [12].

Let $\mathbf{S}_{y}(\Theta)$ be the matrix of sensitivity functions,

$$
\begin{gathered}
\mathbf{S}_{y}(\Theta)=\left(\mathbf{S}_{y}\left(\Theta_{1}\right), \ldots, \mathbf{S}_{y}\left(\Theta_{p}\right)\right) \\
\mathbf{S}_{y}\left(\Theta_{i}\right)^{T}=\left(S_{y}\left(t_{1}, \Theta_{i}\right), \ldots, S_{y}\left(t_{N}, \Theta_{i}\right)\right),
\end{gathered}
$$

where $\mathbf{S}_{y}\left(\Theta_{i}\right) \in \mathbb{R}^{1 \times m N}$ and $\mathbf{S}_{y}(\Theta) \in \mathbb{R}^{p \times m N}$. The practical identifiability can be numerically implemented as a nullrank test of the matrix $\mathbf{S}_{y}(\Theta)$. If the rank of $\mathbf{S}_{y}(\Theta)$ is estimated as significantly null then the model is not locally practically identifiable. The rank of $\mathbf{S}_{y}(\Theta)$ can be viewed as a practical identifiability degree of a model structure for a given experiment.

\section{B. Sensitivity analysis of block diagrams by computer alge- bra}

Equation (9) also emphasizes the crucial role of sensitivity analysis in the local assessment of the practical identifiability. A symbolic approach which eliminates the drawbacks of the finite-difference approximations and the complexity of the automatic differentiation is proposed and implemented into a software: Diffedge ${ }^{(}$(http://www. appedge.com/). It combines a computer algebra system and block diagrams to compute the derivatives of a Simulink model with respect to its independent parameters. The derivative model is also represented by a block diagram and can be used like any Simulink model.

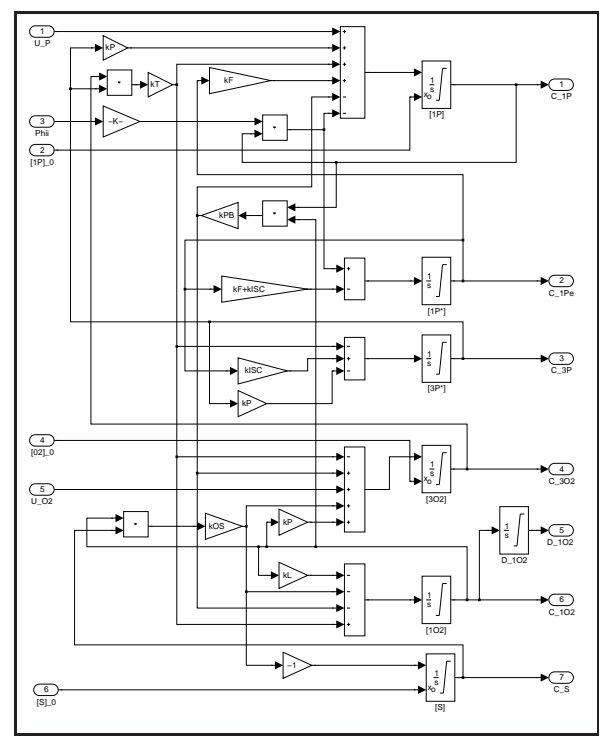

Fig. 1. Block diagram of the photoreactions

\section{Application to the photoreaction model}

Fig. 1 depicts the block diagram of eq.(2) implemented into the simulation environment Simulink ${ }^{\complement}$. The initial values of the states have been chosen from literature [10]. $\Phi_{i}$ is a square signal (width: $300 \mathrm{~s}$ ), $U_{P}=0$, and $U_{O_{2}}=$ $1.5 \cdot 10^{-4} \mathrm{~mol} \cdot L^{-1} \cdot \mathrm{s}^{-1}$. Nominal values of the physical parameters in $\Theta$ correspond to in vitro values obtained in scientific literatures [10], [6]. The numerical $\operatorname{rank}$ of $\mathbf{S}_{y}(\Theta)$ is equal to 7 , therefore all the parameters are practically identifiable.

In conclusion, all the considered parameters can be estimated from a realistic in vivo experiment.

\section{PRACTICAL IDENTIFIABILITY RANKING OF PHOTOPHYSICAL PARAMETERS}

Knowing the number of identifiable parameters, it is also interesting to sort out the parameters according to their influence on $y$ and their cross-correlation of their sensitivity functions. Indeed, more a parameter is practically identifiable, more accurate is its estimate.

\section{A. Output sensitivity ranking}

The first classification consists in sorting out (in a descending order) the parameters according to their influence on the output variable. The comparison criterion proposed herein can be defined as follows

$$
L\left(\Theta_{i}\right)=\left|S_{y}\left(\Theta_{i}\right)\right|=\left|\frac{\partial y(\Theta)}{\partial \Theta_{i}}\right|,
$$

where $S_{y}\left(\Theta_{i}\right)$ denotes the sensitivity function of $y(\Theta)$ with respect to the model parameter $\Theta_{i}$. Results are presented in fig. 2 by a logarithmic diagram. It is shown that $k_{l}$ is the most significant parameter, whereas $k_{T}$ is the least significant parameter, in the sense of the maximization of $L$. 


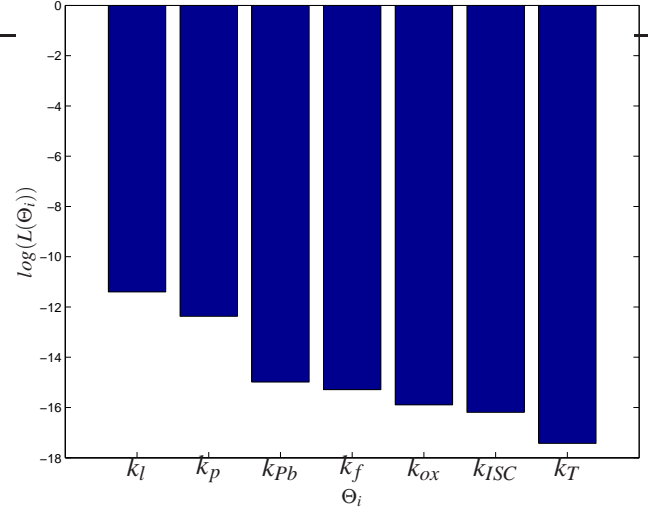

Fig. 2. Output-sensitivity ranking

\section{B. Condition number ranking}

In a second step, a new classification is performed by taking into account the cross-correlation between the sensitivity functions. The vectors $\mathbf{S}_{y}\left(\Theta_{i}\right) \in \mathbb{R}^{N}$ are sorted out according to the condition number of a matrix $\Sigma \in \mathbb{R}^{N \times \mu}$ where $\mu$ is the number of identifiable parameters. $\Sigma$ is iteratively built up from its first column $\Sigma_{1}=S_{y}\left(\Theta_{\operatorname{Lmax}}\right)$ such that

$$
\Theta_{\text {Lmax }}=\arg \max _{\Theta_{i}} L\left(\Theta_{i}\right) \quad i \in\{1, \cdots, \mu\} .
$$

In other words, $\Sigma$ is initialized by the sensitivity fonction of the most influent parameter on the output, i.e. $S_{J}\left(k_{l}\right)$ in this case. The other columns of $\Sigma$ are chosen among the remaining sensitivity functions and are arranged in such an order that

$$
\begin{aligned}
& \Sigma_{j}=S_{y}\left(\check{\Theta}_{i}\right) \quad \text { with: } \\
& \check{\Theta}_{i}=\arg \min _{\Theta_{i}} \operatorname{cond}\left(\Sigma_{1, j}\left(\Theta_{i}\right)\right) \quad \forall \Theta_{i} \neq \Theta_{\text {Lmax }}
\end{aligned}
$$

and $j \in\{2, \cdots, \mu\} . \Sigma_{j}$ denotes the $j^{\text {th }}$ column of $\Sigma$ and $\Sigma_{1, j}\left(\Theta_{i}\right)=\left[\Sigma_{1}, \cdots, \Sigma_{j-1}, S_{J}\left(\Theta_{i}\right)\right]$. Hence, $\Sigma_{j}$ is selected among a given number of sensitivity functions in order to minimize the condition number of $\Sigma_{1, j}$. The final arrangement of the sensitivity functions is described by a spectrum of the condition number of $\Sigma_{1, j}$ with respect to $j$. Fig. 3 shows the spectrum of the condition numbers of $\Sigma_{1, j}$ with respect to $j$. The final ranking of the parameters is given by the $\mathrm{x}$-axis of this spectrum. It appears that $k_{l}$ is the most identifiable parameter and $k_{T}$ is the least one. In other terms, the estimation of $k_{T}$ will be still uncertain than for $k_{l}$.

\section{CONCLUSiOnS}

This article deals with the practical identifiability of the photophysical parameters in PDT. In this study, we have considered realistic in vivo constraints: (i) the input signal is a wide square signal and (ii) there is only one measurement, the concentration of intracellular photosensitiser. In these conditions, it is shown that seven photophysical parameters are identifiable. This result opens new perspectives concerning the estimation of photophysical parameters in PDT. The main benefit of the proposed approach is a significant reduction of the experimental cost. Indeed, the seven photophysical

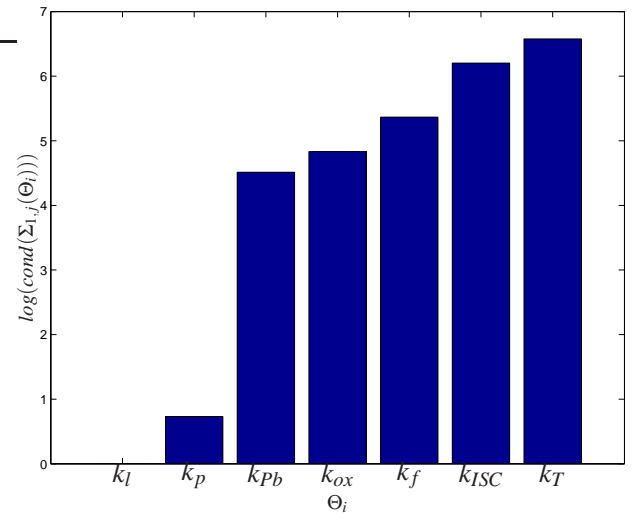

Fig. 3. Condition numbers spectrum of $\Sigma_{1, j}$

parameters are estimated from one 'dynamic' experiment instead of several 'static' ones as it is usually the case for the estimation of quantum yields. The other main advantage of such an approach is to directly estimate the photophysical parameters in in vivo conditions. Future investigations should examine the impact of the input design on the condition number of the sensitivity matrix and new experiments will be handled to estimate some photophysical parameters .

\section{REFERENCES}

[1] J. G. Moser, Photodynamic Tumor Therapy: 2nd and 3rd Generation. Gordon \& Breach Science Publishers, 1998.

[2] M. P. Goldman, Photodynamic Therapy. W.B. Saunders Company, 2005.

[3] M. Niedre, M. S. Patterson, and B. C. Wilson, "Direct near-infrared luminescence detection of singlet oxygen generated by photodynamic therapy in cells in vitro and tissues in vivo," Photochemistry and Photobiology, vol. 75, no. 4, pp. 382-391, 2002.

[4] M. Niedre, C. Yu, M. Patterson, and B. Wilson, "Singlet oxygen luminescence as an in vivo photodynamic therapy dose metric: validation in normal mouse skin with topical amino-levulinic acid," British Journal of Cancer, vol. 92, pp. 298 - 304, 2005.

[5] T. Bastogne, M. Thomassin, and J. Masse, "Selection and identification of physical parameters from passive observation. application to a winding process," Accepted in Control Engineering Practice, 2007.

[6] R. Bonnett, B.D. Djelal, P.A. Hamilton,G. Martinez, F. Wierrani, "Photobleaching of 5,10,15,20-tetrakis(m-hydroxyphenyl)porphyrin (m-THPP) and the corresponding chlorin (m-THPC) and bacteriochlorin(m-THPBC). A comparative study", in Journal of Photochemistry and Photobiology, 1999, (53), pp. 136-143.

[7] A. M. Braun, E. Oliveros, "Applications of singlet oxygen reactions: mechanistic and kinetic investigations", Pure \& Appl. Chem., 1990, (62), pp. 1467-1476.

[8] M. C. DeRosa, R. J. Crutchley, "Photosensitized singlet oxygen and its applications", Coordination Chemistry Reviews, 2002, pp. 351-371.

[9] T. J. Dougherty, C. J. Gomer, B. W. Henderson, G. Jori, D. Kessel, M. Korbelik, J. Moan, Q. Peng, "Photodynamic Therapy", J Natl Cancer Inst., vol. 90, pp. 889 - 905, 1998.

[10] T. Foster, R. S. Murant, R. G. Bryant, R. S. Knox, S. L. Gibson, and R. Hilf, "Oxygen consumption and diffusion effects in photodynamic therapy," Radiation Research, vol. 126, pp. 296-303, 1991.

[11] E. Walter and L. Pronzato, Identification of Parametric Models from experimental data. Springer-Verlag, Masson, 1997.

[12] M. S. Grewal and K. Glover, "Identifiability of linear and nonlinear dynamical systems," IEEE Trans. on Automatic Control, vol. 21, no. 6, pp. 833-837, 1976.

[13] P. Vanrolleghem, M. Van Daele, and D. Dochain, "Practical identifiability of a biokinetic model of activated sludge respiration," Wat. Res., vol. 29, no. 11, pp. 2561-2570, 1995.

[14] D. Dochain and P. Vanrolleghem, Dynamical Modelling and Estimation in Wastewater Treatment Processes. IWA Publishing, 2001. 